1998-01-01

\title{
Performance and robustness issues in the compensation of FOLPD processes with PI and PID controllers
}

\author{
Aidan O'Dwyer \\ Technological University Dublin, aidan.odwyer@tudublin.ie
}

Follow this and additional works at: https://arrow.tudublin.ie/engscheleart

Part of the Controls and Control Theory Commons

\section{Recommended Citation}

O'Dwyer, Aidan : Performance and robustness issues in the compensation of FOLPD processes with PI and PID controllers. Proceedings of the Irish Signals and Systems Conference, pp. 227-234, Dublin Institute of Technology, Kevin St., Dublin, June, 1998. doi:10.21427/zp6m-7e41

This Conference Paper is brought to you for free and open access by the School of Electrical and Electronic Engineering at ARROW@TU Dublin. It has been accepted for inclusion in Conference papers by an authorized administrator of ARROW@TU Dublin. For more information, please contact arrow.admin@tudublin.ie, aisling.coyne@tudublin.ie,gerard.connolly@tudublin.ie.

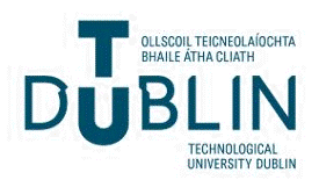




\title{
Performance and robustness issues in the compensation of FOLPD processes with PI and PID controllers
}

\author{
A. O’Dwyer \\ School of Control Systems and Electrical Engineering, \\ Dublin Institute of Technology, \\ Kevin St., \\ Dublin 8.
}

\begin{abstract}
This paper will discuss the compensation of first order lag plus time delay (FOLPD) processes using PI and PID controllers whose parameters are specified using appropriate tuning rules. The gain margin, phase margin and maximum sensitivity of the compensated system as the ratio of time delay to time constant of the process varies, are used as ways of judging the performance and robustness of the system.
\end{abstract}

Keywords: PID, time delay, gain margin, phase margin, maximum sensitivity.

\section{Introduction}

The ability of proportional integral (PI) and proportional integral derivative (PID) controllers to compensate most practical industrial processes has led to their wide acceptance in industrial applications. The requirement to choose either two or three controller parameters has meant that the use of tuning rules to determine these parameters is popular. The author is aware of 72 such tuning rules in the literature to specify the PI controller terms to compensate a FOLPD process (with another 24 tuning rules which are non-process specific, such as the ultimate cycle tuning rule of Ziegler and Nichols [1]), with 116 tuning rules defined to specify the PID controller parameters for FOLPD processes (though it is fair to point out that in the case of the PID controller, separate tuning rules have been defined for a number of PID controller structures). Typical tuning methods are based on using process reaction curve data (e.g. Ziegler and Nichols [1]), integral error criteria (e.g. Rovira et al. [2]), ultimate cycle methods (e.g. Ziegler and Nichols [1]), direct synthesis methods (e.g. Smith and Corripio [3]), gain and phase margin specifications (e.g. Hang et al. [4]) and internal model control strategies (e.g. Morari and Zafiriou [5]).

There has always been some difficulty in objectively comparing the performance and robustness of closed loop compensated systems whose controllers are determined by these tuning rules, as the time delay of the FOLPD process varies. Recently, however, Ho et al. [6], [7] determined performance and robustness by analytically calculating a good approximation for the gain and phase margins of the resulting compensated systems, and applying the technique to consider 11 tuning rules (PI controller) and 10 tuning rules (PID controller). The present paper will apply the method to determine the gain and phase margins of processes compensated by a wider range of PI and PID tuning rules, to facilitate direct comparisons of a wide variety of tuning rules. In addition, the paper will extend the method to determine analytically the maximum sensitivity of the compensated system, as an alternative performance 
and robustness measure. Insight will be obtained into the range of time delay to time constant ratios over which it is sensible to apply various tuning rules, to compensate a FOLPD process.

The paper is organised as follows. Formulae for calculating analytically the performance and robustness metrics are outlined in Section 2. The performance and robustness of FOLPD processes compensated with a wide variety of PI and PID tuning rules are evaluated in Section 3. In Section 4, conclusions of the work are provided.

\section{The analytical determination of performance and robustness metrics}

\subsection{The calculation of gain and phase margins}

The calculations of the gain and phase margins of systems compensated by both PI and PID controllers are presented. Most of this information also appears in Ho et al. [6], [7].

\subsubsection{PI tuning formulae}

The process and controller transfer functions are denoted by $G_{m}(s)$ and $G_{c}(s)$, respectively, and the gain and phase margins as $\mathrm{A}_{\mathrm{m}}$ and $\phi_{\mathrm{m}}$, respectively. From the definition of gain and phase margin, the following sets of equations are obtained:

$$
\phi_{\mathrm{m}}=\arg \left[\mathrm{G}_{\mathrm{c}}\left(\mathrm{j} \omega_{\mathrm{g}}\right) \mathrm{G}_{\mathrm{m}}\left(\mathrm{j} \omega_{\mathrm{g}}\right)\right]+\pi
$$

$$
A_{m}=\frac{1}{\left|G_{c}\left(j \omega_{p}\right) G_{m}\left(j \omega_{p}\right)\right|}
$$

where $\omega_{\mathrm{g}}$ and $\omega_{\mathrm{p}}$ are given by

$$
\left|G_{c}\left(j \omega_{g}\right) G_{m}\left(j \omega_{g}\right)\right|=1
$$

$$
\arg \left[G_{c}\left(j \omega_{p}\right) G_{m}\left(j \omega_{p}\right)\right]=-\pi
$$

The controller and process model are respectively given by

$$
\mathrm{G}_{\mathrm{c}}(\mathrm{s})=\mathrm{K}_{\mathrm{c}}\left(1+\frac{1}{\mathrm{~T}_{\mathrm{i}} \mathrm{s}}\right)
$$

$$
\mathrm{G}_{\mathrm{m}}(\mathrm{s})=\frac{\mathrm{K}_{\mathrm{m}} \mathrm{e}^{-\mathrm{s \tau} \mathrm{m}}}{1+\mathrm{s} \mathrm{T}_{\mathrm{m}}}
$$

Substituting equations (3a) and (3b) into equations (1a) to (2b) gives

$$
\phi_{\mathrm{m}}=0.5 \pi+\tan ^{-1} \omega_{\mathrm{g}} \mathrm{T}_{\mathrm{i}}-\tan ^{-1} \omega_{\mathrm{g}} \mathrm{T}_{\mathrm{m}}-\omega_{\mathrm{g}} \tau_{\mathrm{m}} \text { (4a) } \quad \mathrm{A}_{\mathrm{m}}=\frac{\omega_{\mathrm{p}} \mathrm{T}_{\mathrm{i}}}{\mathrm{K}_{\mathrm{c}} \mathrm{K}_{\mathrm{m}}} \sqrt{\frac{\omega_{\mathrm{p}}{ }^{2} \mathrm{~T}_{\mathrm{m}}{ }^{2}+1}{\omega_{\mathrm{p}}{ }^{2} \mathrm{~T}_{\mathrm{i}}^{2}+1}}
$$

From equation (2a), $\omega_{\mathrm{g}}$ may be determined analytically to be

$$
\omega_{\mathrm{g}}=\sqrt{\frac{\mathrm{T}_{\mathrm{i}}\left(\mathrm{K}_{\mathrm{c}}{ }^{2} \mathrm{~K}_{\mathrm{m}}{ }^{2}-1\right)+\sqrt{\left(\mathrm{K}_{\mathrm{c}}{ }^{2} \mathrm{~K}_{\mathrm{m}}{ }^{2}-1\right)^{2} \mathrm{~T}_{\mathrm{i}}{ }^{2}+4 \mathrm{~K}_{\mathrm{c}}{ }^{2} \mathrm{~K}_{\mathrm{m}}{ }^{2} \mathrm{~T}_{\mathrm{m}}{ }^{2}}}{2 \mathrm{~T}_{\mathrm{i}} \mathrm{T}_{\mathrm{m}}{ }^{2}}}
$$

From equation $(2 b), \omega_{p}$ is given by

$$
0.5 \pi+\tan ^{-1} \omega_{\mathrm{p}} \mathrm{T}_{\mathrm{i}}-\tan ^{-1} \omega_{\mathrm{p}} \mathrm{T}_{\mathrm{m}}-\omega_{\mathrm{p}} \tau_{\mathrm{m}}=0
$$


An analytical solution of this equation is not possible. An approximate analytical solution may be obtained if the following approximation for the arctan function is made:

$$
\tan ^{-1} \mathrm{x} \approx \frac{\pi}{4} \mathrm{x},|\mathrm{x}|<1 \text { and } \tan ^{-1} \mathrm{x} \approx \frac{\pi}{2}-\frac{\pi}{4 \mathrm{x}},|\mathrm{x}|>1
$$

This is quite an accurate approximation, as is shown by Ho et al. [6] Looking at equation (6), four possibilities present themselves if the approximation in equation (7) is to be used; these possibilities are (i) $\omega_{\mathrm{p}} \mathrm{T}_{\mathrm{i}}>1, \omega_{\mathrm{p}} \mathrm{T}_{\mathrm{m}}>1$ (ii) $\omega_{\mathrm{p}} \mathrm{T}_{\mathrm{i}}>1, \omega_{\mathrm{p}} \mathrm{T}_{\mathrm{m}}<1$ (iii) $\omega_{\mathrm{p}} \mathrm{T}_{\mathrm{i}}<1, \omega_{\mathrm{p}} \mathrm{T}_{\mathrm{m}}>1$ and (iv) $\omega_{\mathrm{p}} \mathrm{T}_{\mathrm{i}}<1, \omega_{\mathrm{p}} \mathrm{T}_{\mathrm{m}}<1$ (Ho et al. [6] consider just the first of these possibilities). Table 1 shows the formula for $\omega_{\mathrm{p}}$ that may be determined analytically for each of these cases.

Table 1: Formulae for $\omega_{\mathrm{p}}$

\begin{tabular}{|c|c|c|c|}
\hline$\omega_{\mathrm{p}} \mathrm{T}_{\mathrm{i}}>1, \omega_{\mathrm{p}} \mathrm{T}_{\mathrm{m}}>1$ & $\omega_{\mathrm{p}} \mathrm{T}_{\mathrm{i}}>1, \omega_{\mathrm{p}} \mathrm{T}_{\mathrm{m}}<1$ & $\omega_{\mathrm{p}} \mathrm{T}_{\mathrm{i}}<1, \omega_{\mathrm{p}} \mathrm{T}_{\mathrm{m}}>1$ & $\omega_{\mathrm{p}} \mathrm{T}_{\mathrm{i}}<1, \omega_{\mathrm{p}} \mathrm{T}_{\mathrm{m}}<1$ \\
\hline$\omega_{\mathrm{p}}=\frac{\pi \pm \sqrt{\pi^{2}-4 \pi \tau_{\mathrm{m}}\left(\frac{1}{\mathrm{~T}_{\mathrm{i}}}-\frac{1}{\mathrm{~T}_{\mathrm{m}}}\right)}}{4 \tau_{\mathrm{m}}}$ & $\omega_{\mathrm{p}}=\frac{\pi \pm \sqrt{\pi^{2}-\frac{\pi}{\mathrm{T}_{\mathrm{i}}}\left(\frac{\pi}{4} \mathrm{~T}_{\mathrm{m}}+\tau_{\mathrm{m}}\right)}}{2\left(\frac{\pi}{4} \mathrm{~T}_{\mathrm{m}}+\tau_{\mathrm{m}}\right)}$ & $\omega_{\mathrm{p}}=\sqrt{\frac{\pi / \mathrm{T}_{\mathrm{m}}}{4 \tau_{\mathrm{m}}-\pi \mathrm{T}_{\mathrm{i}}}}$ & $\omega_{\mathrm{p}}=\frac{2 \pi}{4 \tau_{\mathrm{m}}+\pi\left(\mathrm{T}_{\mathrm{m}}-\mathrm{T}_{\mathrm{i}}\right)}$ \\
\hline
\end{tabular}

Equations (4a) and (4b), together with equation (5) and the relevant equations from Table 1, may now be used to calculate the gain and phase margin of the compensated system, for each of the tuning rules, as a function of $\tau_{\mathrm{m}} / \mathrm{T}_{\mathrm{m}}$.

\subsubsection{PID tuning formulae}

The controller is given by

$$
\mathrm{G}_{\mathrm{c}}(\mathrm{s})=\mathrm{K}_{\mathrm{c}}\left(1+\frac{1}{\mathrm{~T}_{\mathrm{i}} \mathrm{s}}\right)\left(\frac{1+\mathrm{sT}}{1+\mathrm{s} \alpha \mathrm{T}_{\mathrm{d}}}\right)
$$

and the process model is given by equation (3b). Substituting equations (3b) and (8) into equations (1a) to (2b) gives

$$
\phi_{\mathrm{m}}=0.5 \pi+\tan ^{-1} \omega_{\mathrm{g}} \mathrm{T}_{\mathrm{i}}+\tan ^{-1} \omega_{\mathrm{g}} \mathrm{T}_{\mathrm{d}}-\tan ^{-1} \omega_{\mathrm{g}} \mathrm{T}_{\mathrm{m}}-\tan ^{-1} \omega_{\mathrm{g}} \alpha \mathrm{T}_{\mathrm{d}}-\omega_{\mathrm{g}} \tau_{\mathrm{m}}
$$

and

$$
A_{m}=\frac{\omega_{p} T_{i}}{K_{c} K_{m}} \sqrt{\frac{\left(1+\omega_{p}^{2} T_{m}^{2}\right)\left(1+\omega_{p}^{2} \alpha^{2} T_{d}^{2}\right)}{\left(1+\omega_{p}{ }^{2} T_{i}^{2}\right)\left(1+\omega_{p}{ }^{2} T_{d}^{2}\right)}}
$$

From equation $(2 \mathrm{a}), \omega_{\mathrm{g}}$ is given by the solution of

$$
\omega_{\mathrm{g}}{ }^{6}+\mathrm{a}_{1} \omega_{\mathrm{g}}{ }^{4}+\mathrm{a}_{2} \omega_{\mathrm{g}}{ }^{2}+\mathrm{a}_{3}=0
$$

with $\mathrm{a}_{1}=\frac{\mathrm{T}_{\mathrm{i}}^{2}\left(\mathrm{~T}_{\mathrm{m}}{ }^{2}+\alpha^{2} \mathrm{~T}_{\mathrm{d}}{ }^{2}\right)-\mathrm{K}_{\mathrm{c}}{ }^{2} \mathrm{~K}_{\mathrm{m}}{ }^{2} \mathrm{~T}_{\mathrm{i}}^{2} \mathrm{~T}_{\mathrm{d}}{ }^{2}}{\mathrm{~T}_{\mathrm{i}}{ }^{2} \mathrm{~T}_{\mathrm{m}}{ }^{2} \alpha^{2} \mathrm{~T}_{\mathrm{d}}{ }^{2}}, \mathrm{a}_{2}=\frac{\mathrm{T}_{\mathrm{i}}{ }^{2}-\mathrm{K}_{\mathrm{c}}{ }^{2} \mathrm{~K}_{\mathrm{m}}{ }^{2}\left(\mathrm{~T}_{\mathrm{i}}{ }^{2}+\mathrm{T}_{\mathrm{d}}{ }^{2}\right)}{\mathrm{T}_{\mathrm{i}}{ }^{2} \mathrm{~T}_{\mathrm{m}}{ }^{2} \alpha^{2} \mathrm{~T}_{\mathrm{d}}{ }^{2}}$ and $\mathrm{a}_{3}=-\frac{\mathrm{K}_{\mathrm{c}}{ }^{2} \mathrm{~K}_{\mathrm{m}}{ }^{2}}{\mathrm{~T}_{\mathrm{i}} \mathrm{T}_{\mathrm{m}}{ }^{2} \alpha^{2} \mathrm{~T}_{\mathrm{d}}{ }^{2}}$.

Following the procedure outlined by Ho et al. [7], the analytical solution of equation (11) is given as 


$$
\omega_{g}=\sqrt{\sqrt[3]{\mathrm{R}+\sqrt{\mathrm{Q}^{3}+\mathrm{R}^{2}}}+\sqrt[3]{\mathrm{R}-\sqrt{\mathrm{Q}^{3}+\mathrm{R}^{2}}}-\frac{\mathrm{a}_{1}}{3}}
$$

with $\mathrm{Q}=\frac{3 \mathrm{a}_{2}-\mathrm{a}_{1}^{2}}{9}$ and $\mathrm{R}=\frac{9 \mathrm{a}_{1} \mathrm{a}_{2}-27 \mathrm{a}_{3}-2 \mathrm{a}_{1}{ }^{3}}{54}$

From equation $(2 b), \omega_{\mathrm{p}}$ is given by

$$
0.5 \pi+\tan ^{-1} \omega_{\mathrm{p}} \mathrm{T}_{\mathrm{d}}+\tan ^{-1} \omega_{\mathrm{p}} \mathrm{T}_{\mathrm{i}}-\tan ^{-1} \omega_{\mathrm{p}} \mathrm{T}_{\mathrm{m}}-\tan ^{-1} \omega_{\mathrm{p}} \alpha \mathrm{T}_{\mathrm{d}}-\omega_{\mathrm{p}} \tau_{\mathrm{m}}=0
$$

As in Section 2.1.1, an analytical solution of this equation is not possible. An approximate analytical solution may be obtained if the approximation detailed in equation (7) is made. Looking at equation (13), twelve possibilities present themselves if the approximation in equation (7) is to be used; these possibilities are
(1) $\omega_{\mathrm{p}} \mathrm{T}_{\mathrm{i}}>1, \omega_{\mathrm{p}} \mathrm{T}_{\mathrm{m}}>1$
(2) $\omega_{\mathrm{p}} \mathrm{T}_{\mathrm{i}}>1, \quad \omega_{\mathrm{p}} \mathrm{T}_{\mathrm{m}}<1$
(3) $\omega_{\mathrm{p}} \mathrm{T}_{\mathrm{i}}<1, \omega_{\mathrm{p}} \mathrm{T}_{\mathrm{m}}>1$
(4) $\omega_{\mathrm{p}} \mathrm{T}_{\mathrm{i}}<1, \omega_{\mathrm{p}} \mathrm{T}_{\mathrm{m}}<1$
(a) $\overline{\omega_{\mathrm{p}} \mathrm{T}_{\mathrm{d}} \leq 1, \omega_{\mathrm{p}} \alpha \mathrm{T}_{\mathrm{d}}<1}$
(a) $\omega_{\mathrm{p}} \mathrm{T}_{\mathrm{d}} \leq 1, \omega_{\mathrm{p}} \alpha \mathrm{T}_{\mathrm{d}}<1$
(a) $\omega_{\mathrm{p}} \mathrm{T}_{\mathrm{d}} \leq 1, \omega_{\mathrm{p}} \alpha \mathrm{T}_{\mathrm{d}}<1$
(a) $\overline{\omega_{\mathrm{p}} \mathrm{T}_{\mathrm{d}} \leq 1, \omega_{\mathrm{p}} \alpha \mathrm{T}_{\mathrm{d}}<1}$
(b) $\omega_{\mathrm{p}} \mathrm{T}_{\mathrm{d}}>1, \omega_{\mathrm{p}} \alpha \mathrm{T}_{\mathrm{d}}<1$
(b) $\omega_{\mathrm{p}} \mathrm{T}_{\mathrm{d}}>1, \omega_{\mathrm{p}} \alpha \mathrm{T}_{\mathrm{d}}<1$
(b) $\omega_{\mathrm{p}} \mathrm{T}_{\mathrm{d}}>1, \omega_{\mathrm{p}} \alpha \mathrm{T}_{\mathrm{d}}<1$
(b) $\omega_{\mathrm{p}} \mathrm{T}_{\mathrm{d}}>1, \omega_{\mathrm{p}} \alpha \mathrm{T}_{\mathrm{d}}<1$
(c) $\omega_{\mathrm{p}} \mathrm{T}_{\mathrm{d}}>1, \omega_{\mathrm{p}} \alpha \mathrm{T}_{\mathrm{d}}>1$
(c) $\omega_{\mathrm{p}} \mathrm{T}_{\mathrm{d}}>1, \omega_{\mathrm{p}} \alpha \mathrm{T}_{\mathrm{d}}>1$
(c) $\omega_{\mathrm{p}} \mathrm{T}_{\mathrm{d}}>1, \omega_{\mathrm{p}} \alpha \mathrm{T}_{\mathrm{d}}>1$
(c) $\omega_{\mathrm{p}} \mathrm{T}_{\mathrm{d}}>1, \omega_{\mathrm{p}} \alpha \mathrm{T}_{\mathrm{d}}>1$

It is interesting that Ho et al. [7] considers just the case where $\omega_{\mathrm{p}} \mathrm{T}_{\mathrm{i}}>1$ and $\omega_{\mathrm{p}} \mathrm{T}_{\mathrm{m}}>1$. Table 2 shows the formula for $\omega_{\mathrm{p}}$ that may be determined for all of the cases above.

\begin{tabular}{|c|c|c|c|}
\hline & $\omega_{\mathrm{p}} \mathrm{T}_{\mathrm{d}} \leq 1, \quad \omega_{\mathrm{p}} \alpha \mathrm{T}_{\mathrm{d}}<1$ & $\omega_{\mathrm{p}} \mathrm{T}_{\mathrm{d}}>1, \omega_{\mathrm{p}} \alpha \mathrm{T}_{\mathrm{d}}<1$ & $\omega_{\mathrm{p}} \mathrm{T}_{\mathrm{d}}>1, \omega_{\mathrm{p}} \alpha \mathrm{T}_{\mathrm{d}}>1$ \\
\hline $\begin{array}{l}\omega_{\mathrm{p}} \mathrm{T}_{\mathrm{i}}>1, \\
\omega_{\mathrm{p}} \mathrm{T}_{\mathrm{m}}>1\end{array}$ & $\frac{\pi \pm \sqrt{\pi^{2}-\pi\left(4 \tau_{\mathrm{m}}-\pi \mathrm{T}_{\mathrm{d}}(1-\alpha)\right)\left(\frac{1}{\mathrm{~T}_{\mathrm{i}}}-\frac{1}{\mathrm{~T}_{\mathrm{m}}}\right)}}{4 \tau_{\mathrm{m}}-\pi \mathrm{T}_{\mathrm{d}}(1-\alpha)}$ & $\frac{2 \pi \pm \sqrt{4 \pi^{2}-\pi\left(4 \tau_{\mathrm{m}}+\pi \mathrm{T}_{\mathrm{d}} \alpha\right)\left(\frac{1}{\mathrm{~T}_{\mathrm{d}}}+\frac{1}{\mathrm{~T}_{\mathrm{i}}}-\frac{1}{\mathrm{~T}_{\mathrm{m}}}\right)}}{4 \tau_{\mathrm{m}}+\pi \mathrm{T}_{\mathrm{d}} \alpha}$ & $\frac{\pi \pm \sqrt{\pi^{2}-4 \pi \tau_{\mathrm{m}}\left(\frac{1}{T_{\mathrm{d}}}+\frac{1}{\mathrm{~T}_{\mathrm{i}}}-\frac{1}{\alpha \mathrm{T}_{\mathrm{d}}}-\frac{1}{\mathrm{~T}_{\mathrm{m}}}\right)}}{4 \tau_{\mathrm{m}}}$ \\
\hline $\begin{array}{l}\omega_{\mathrm{p}} \mathrm{T}_{\mathrm{i}}>1, \\
\omega_{\mathrm{p}} \mathrm{T}_{\mathrm{m}}<1\end{array}$ & $\frac{2 \pi \pm \sqrt{4 \pi^{2}+\frac{\pi}{T_{i}}\left(\pi T_{d}-\pi T_{m}-\pi \alpha T_{d}-4 \tau_{m}\right)}}{\pi T_{m}+4 \tau_{m}+\pi \alpha T_{d}-\pi T_{d}}$ & $\frac{3 \pi \pm \sqrt{9 \pi^{2}-\pi\left(\frac{1}{T_{d}}+\frac{1}{T_{i}}\right)\left(\pi T_{m}+\pi \alpha T_{d}+4 \tau_{m}\right)}}{\pi T_{m}+4 \tau_{m}+\pi \alpha T_{d}}$ & $\frac{2 \pi \pm \sqrt{4 \pi^{2}+\pi\left(\frac{1}{\alpha T_{d}}-\frac{1}{T_{d}}-\frac{1}{T_{\mathrm{i}}}\right)\left(\pi T_{\mathrm{m}}+4 \tau_{\mathrm{m}}\right)}}{\pi \mathrm{T}_{\mathrm{m}}+4 \tau_{\mathrm{m}}}$ \\
\hline $\begin{array}{l}\omega_{\mathrm{p}} \mathrm{T}_{\mathrm{i}}<1, \\
\omega_{\mathrm{p}} \mathrm{T}_{\mathrm{m}}>1\end{array}$ & $\sqrt{\frac{\pi}{\mathrm{T}_{\mathrm{m}}\left(4 \tau_{\mathrm{m}}+\pi\left[\alpha \mathrm{T}_{\mathrm{d}}-\mathrm{T}_{\mathrm{d}}-\mathrm{T}_{\mathrm{i}}\right]\right)}}$ & $\frac{-\pi \pm \sqrt{\pi^{2}-\pi\left(\frac{1}{T_{m}}-\frac{1}{T_{d}}\right)\left(\pi T_{i}-\pi \alpha T_{d}-4 \tau_{m}\right)}}{\pi T_{i}-4 \tau_{m}-\pi \alpha T_{d}}$ & $\sqrt{\frac{\frac{\pi}{\mathrm{T}_{\mathrm{d}}}-\frac{\pi}{\mathrm{T}_{\mathrm{m}}}-\frac{\pi}{\alpha \mathrm{T}_{\mathrm{d}}}}{\pi \mathrm{T}_{\mathrm{i}}-4 \tau_{\mathrm{m}}}}$ \\
\hline $\begin{array}{l}\omega_{\mathrm{p}} \mathrm{T}_{\mathrm{i}}<1, \\
\omega_{\mathrm{p}} \mathrm{T}_{\mathrm{m}}<1\end{array}$ & $\frac{2 \pi}{\pi\left(\alpha \mathrm{T}_{\mathrm{d}}-\mathrm{T}_{\mathrm{d}}\right)+\pi\left(\mathrm{T}_{\mathrm{m}}-\mathrm{T}_{\mathrm{i}}\right)+4 \tau_{\mathrm{m}}}$ & $\frac{2 \pi \pm \sqrt{4 \pi^{2}-\frac{\pi}{T_{d}}\left(\pi \mathrm{T}_{\mathrm{m}}-\pi \mathrm{T}_{\mathrm{i}}+\pi \alpha \mathrm{T}_{\mathrm{d}}+4 \tau_{\mathrm{m}}\right)}}{\pi \mathrm{T}_{\mathrm{m}}-\pi \mathrm{T}_{\mathrm{i}}+4 \tau_{\mathrm{m}}-\pi \alpha \mathrm{T}_{\mathrm{d}}}$ & $\frac{\pi \pm \sqrt{\pi^{2}-\pi\left(\frac{1}{\alpha T_{d}}-\frac{1}{T_{d}}\right)\left(\pi T_{i}-\pi T_{m}-4 \tau_{m}\right)}}{4 \tau_{m}-\pi T_{m}-\pi T_{i}}$ \\
\hline
\end{tabular}

Table 2: Formulae for $\omega_{\mathrm{p}}$

Equations (9) and (10), together with equation (12) and the relevant equations in Table 2, are now used to calculate the gain and phase margin of the compensated system, for each of the tuning rules, as a function of $\tau_{\mathrm{m}} / \mathrm{T}_{\mathrm{m}}$.

\subsection{The calculation of the maximum sensitivity}

The maximum sensitivity is the shortest distance from the Nyquist curve to the $(-1,0)$ point on the Rl-Im axis. It is defined as follows:

$$
M_{E}=\underset{\text { all } \omega}{\operatorname{Max}}\left|\frac{1}{1+G_{p}(j \omega) G_{c}(j \omega)}\right|
$$


Proceedings of the Irish Signals and Systems Conference, Dublin Institute of Technology, Kevin St., Dublin, Ireland, June, pp. 227-234.

For a FOLPD process model controlled by a PI controller,

$$
\left|G_{p}(j \omega) G_{c}(j \omega)\right|=\frac{\sqrt{1+\omega^{2} T_{i}^{2}}}{\sqrt{1+\omega^{2} T_{m}^{2}}} \frac{K_{c} K_{m}}{\omega T_{i}}
$$

and

$$
\arg \left[G_{p}(j \omega) G_{c}(j \omega)\right]=-0.5 \pi-\tan ^{-1} \omega T_{m}+\tan ^{-1} \omega T_{i}-\omega \tau_{m}
$$

For a FOLPD process model controlled by a PID controller,

$$
\left|G_{p}(j \omega) G_{c}(j \omega)\right|=\frac{\sqrt{\left(1+\omega^{2} T_{i}^{2}\right)\left(1+\omega^{2} T_{d}^{2}\right)}}{\sqrt{\left(1+\omega^{2} T_{m}^{2}\right)\left(1+\alpha^{2} \omega^{2} T_{d}^{2}\right)}} \frac{K_{c} K_{m}}{\omega T_{i}}
$$

and

$$
\arg \left[G_{p}(j \omega) G_{c}(j \omega)\right]=-0.5 \pi-\tan ^{-1} \omega T_{m}-\tan ^{-1} \omega \alpha T_{d}+\tan ^{-1} \omega T_{i}+\tan ^{-1} \omega T_{d}-\omega \tau_{m}
$$

The maximum sensitivity may be calculated over an appropriate range of frequencies corresponding to phase lags of $100^{\circ}$ to $260^{\circ}$.

\section{Simulation results}

Space considerations dictate that only representative simulation results may be provided. In these results, gain margin, phase margin and maximum sensitivity are calculated

\begin{tabular}{|c|c|c|c|c|c|}
\hline & \multicolumn{2}{|c|}{ PI tuning rules } & \multicolumn{3}{|c|}{ PID tuning rules } \\
\hline Rule & $\mathrm{K}_{\mathrm{c}}$ & $\mathrm{T}_{\mathrm{i}}$ & $\mathrm{K}_{\mathrm{c}}$ & $\mathrm{T}_{\mathrm{i}}$ & $\mathrm{T}_{\mathrm{d}}$ \\
\hline $\begin{array}{c}\text { Ziegler and } \\
\text { Nichols [1] } \\
\text { process reaction }\end{array}$ & $\frac{0.9 \mathrm{~T}_{\mathrm{m}}}{\mathrm{K}_{\mathrm{m}} \tau_{\mathrm{m}}}$ & $3.33 \tau_{\mathrm{m}}$ & {$\left[\frac{1.2 \mathrm{~T}_{\mathrm{m}}}{\mathrm{K}_{\mathrm{m}} \tau_{\mathrm{m}}}, \frac{2 \mathrm{~T}_{\mathrm{m}}}{\mathrm{K}_{\mathrm{m}} \tau_{\mathrm{m}}}\right]$} & $2 \tau_{m}$ & $0.5 \tau_{m}$ \\
\hline $\begin{array}{c}\text { Regulator - IAE } \\
\text { PI - Murrill [8], } \\
\text { IAE PID - Kaya } \\
\text { and Scheib [9] }\end{array}$ & $\frac{0.984}{\mathrm{~K}_{\mathrm{m}}}\left(\frac{\mathrm{T}_{\mathrm{m}}}{\tau_{\mathrm{m}}}\right)^{0.986}$ & $\frac{\mathrm{T}_{\mathrm{m}}}{0.608}\left(\frac{\tau_{\mathrm{m}}}{\mathrm{T}_{\mathrm{m}}}\right)^{0.707}$ & $\frac{0.98089}{\mathrm{~K}_{\mathrm{m}}}\left(\frac{\mathrm{T}_{\mathrm{m}}}{\tau_{\mathrm{m}}}\right)^{0.76167}$ & $\frac{\mathrm{T}_{\mathrm{m}}}{0.91032}\left(\frac{\mathrm{T}_{\mathrm{m}}}{\tau_{\mathrm{m}}}\right)^{1.05221}$ & $0.59974 \mathrm{~T}_{\mathrm{m}}\left(\frac{\tau_{\mathrm{m}}}{\mathrm{T}_{\mathrm{m}}}\right)$ \\
\hline $\begin{array}{c}\text { Regulator - ISE } \\
\text { PI - Murrill [8], } \\
\text { ISE PID - Kaya } \\
\text { and Scheib [9] }\end{array}$ & $\frac{1.305}{\mathrm{~K}_{\mathrm{m}}}\left(\frac{\mathrm{T}_{\mathrm{m}}}{\tau_{\mathrm{m}}}\right)^{0.959}$ & $\frac{\mathrm{T}_{\mathrm{m}}}{0.492}\left(\frac{\tau_{\mathrm{m}}}{\mathrm{T}_{\mathrm{m}}}\right)^{0.739}$ & $\frac{1.11907}{\mathrm{~K}_{\mathrm{m}}}\left(\frac{\mathrm{T}_{\mathrm{m}}}{\tau_{\mathrm{m}}}\right)^{0.89711}$ & $\frac{\mathrm{T}_{\mathrm{m}}}{0.7987}\left(\frac{\mathrm{T}_{\mathrm{m}}}{\tau_{\mathrm{m}}}\right)^{0.9548}$ & $0.54766 \mathrm{~T}_{\mathrm{m}}\left(\frac{\tau_{\mathrm{m}}}{\mathrm{T}_{\mathrm{m}}}\right)$ \\
\hline \begin{tabular}{|} 
Regulator - ITAE \\
PI - Murrill [8], \\
ITAE PID - Kaya \\
and Scheib [9]
\end{tabular} & $\frac{0.859}{\mathrm{~K}_{\mathrm{m}}}\left(\frac{\mathrm{T}_{\mathrm{m}}}{\tau_{\mathrm{m}}}\right)^{0.977}$ & $\frac{\mathrm{T}_{\mathrm{m}}}{0.674}\left(\frac{\tau_{\mathrm{m}}}{\mathrm{T}_{\mathrm{m}}}\right)^{0.680}$ & $\frac{0.77902}{\mathrm{~K}_{\mathrm{m}}}\left(\frac{\mathrm{T}_{\mathrm{m}}}{\tau_{\mathrm{m}}}\right)^{1.0640}$ & $\frac{\mathrm{T}_{\mathrm{m}}}{1.14311}\left(\frac{\mathrm{T}_{\mathrm{m}}}{\tau_{\mathrm{m}}}\right)^{0.70949}$ & $0.57137 \mathrm{~T}_{\mathrm{m}}\left(\frac{\tau_{\mathrm{m}}}{\mathrm{T}_{\mathrm{m}}}\right)^{1}$ \\
\hline $\begin{array}{c}\text { Ziegler and } \\
\text { Nichols [1] }\end{array}$ & $0.45 \mathrm{~K}_{\mathrm{u}}$ & $0.83 \mathrm{~T}_{\mathrm{u}}$ & {$\left[0.6 \mathrm{~K}_{\mathrm{u}}, \mathrm{K}_{\mathrm{u}}\right]$} & $0.5 T_{u}$ & $0.125 T_{u}$ \\
\hline $\begin{array}{c}\text { Astrom and } \\
\text { Hagglund [10] }\end{array}$ & $0.4698 \mathrm{~K}_{\mathrm{u}}$ & $0.4373 \mathrm{~T}_{\mathrm{u}}$ & $0.4698 \mathrm{~K}_{\mathrm{u}}$ & $0.4546 \mathrm{~T}_{\mathrm{u}}$ & $0.1136 \mathrm{~T}_{\mathrm{u}}$ \\
\hline $\begin{array}{c}\text { Servo - Zhuang } \\
\text { and Atherton } \\
{[11]}\end{array}$ & $0.361 \mathrm{~K}_{\mathrm{u}}$ & $0.083\left(1.935 \mathrm{~K}_{\mathrm{m}} \mathrm{K}_{\mathrm{u}}+1\right) \mathrm{T}_{\mathrm{u}}$ & $0.509 \mathrm{~K}_{\mathrm{u}}$ & $0.051\left(3.302 \mathrm{~K}_{\mathrm{m}} \mathrm{K}_{\mathrm{u}}+1\right) \mathrm{T}_{\mathrm{u}}$ & $0.125 \mathrm{~T}_{\mathrm{u}}$ \\
\hline
\end{tabular}
for processes compensated using a PI or PID controller following the tuning rules provided in Table 3.

Table 3: Tuning rules

${ }^{1} \mathrm{~K}_{\mathrm{u}}$ and $\mathrm{T}_{\mathrm{u}}$ are the ultimate gain and ultimate period of the FOLPD model. 
Proceedings of the Irish Signals and Systems Conference, Dublin Institute of Technology, Kevin St., Dublin, Ireland, June, pp. 227-234.

Table 3 (continued)

\begin{tabular}{|c|c|c|c|c|c|}
\hline & \multicolumn{2}{|c|}{ PI tuning rules } & \multicolumn{3}{|c|}{ PID tuning rules } \\
\hline Rule & $\mathrm{K}_{\mathrm{c}}$ & $\mathrm{T}_{\mathrm{i}}$ & $\mathrm{K}_{\mathrm{c}}$ & $\mathrm{T}_{\mathrm{i}}$ & $\mathrm{T}_{\mathrm{d}}$ \\
\hline $\begin{array}{c}\text { Regulator - } \\
\text { Zhuang and } \\
\text { Atherton [11] }\end{array}$ & $\left(\frac{1.892 \mathrm{~K}_{\mathrm{m}} \mathrm{K}_{\mathrm{u}}+0.244}{3.249 \mathrm{~K}_{\mathrm{m}} \mathrm{K}_{\mathrm{u}}+2.097}\right) \mathrm{K}_{\mathrm{u}}$ & $\left(\frac{0.706 \mathrm{~K}_{\mathrm{m}} \mathrm{K}_{\mathrm{u}}-0.227}{0.7229 \mathrm{~K}_{\mathrm{m}} \mathrm{K}_{\mathrm{u}}+1.2736}\right) \mathrm{K}$ & $\frac{4.434 \mathrm{~K}_{\mathrm{m}} \mathrm{K}_{\mathrm{u}}-0.966}{5.12 \mathrm{~K}_{\mathrm{m}} \mathrm{K}_{\mathrm{u}}+1.734} \mathrm{~K}_{\mathrm{u}}$ & $\frac{1.751 \mathrm{~K}_{\mathrm{m}} \mathrm{K}_{\mathrm{u}}-0.612}{3.776 \mathrm{~K}_{\mathrm{m}} \mathrm{K}_{\mathrm{u}}+1.388} \mathrm{~T}_{\mathrm{u}}$ & $0.144 \mathrm{~T}_{\mathrm{u}}$ \\
\hline
\end{tabular}

The MATLAB package has been used in the simulations. It should be stated that $\alpha$ in Equation (8) is taken as 0.1, with any tuning rules for the parallel form of the PID controller being converted into the PID controller structure used, following the procedure of Ho et al. [7]. Figures 1 to 6 show how gain margin, phase margin and maximum sensitivity vary as the ratio of time delay to time constant varies, if some PI tuning rules are used (Figures 1 to 3 ) and corresponding PID tuning rules are used (Figures 4 to 6). In these results, Z-N refers to the process reaction curve method of Ziegler and Nichols [1] with IAE reg, ISE reg and ITAE reg referring to the tuning rules for regulator applications that minimise the integral of absolute error criterion, the integral of squared error criterion and the integral of time multiplied by absolute error criterion, respectively, as defined by Murrill [8] for PI tuning rules and Kaya and Scheib [9] for PID tuning rules.

Figure 1: Gain margin

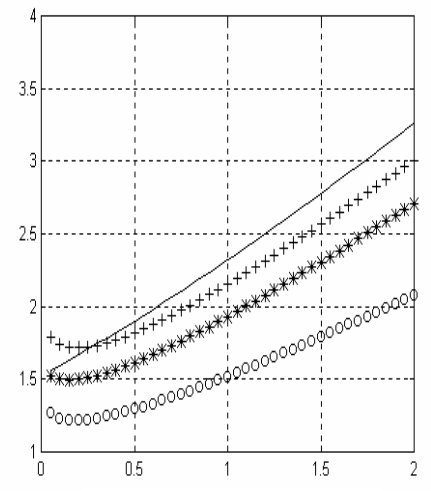

Ratio of $\tau_{\mathrm{m}}$ to $\mathrm{T}_{\mathrm{m}}$

Figure 4: Gain margin

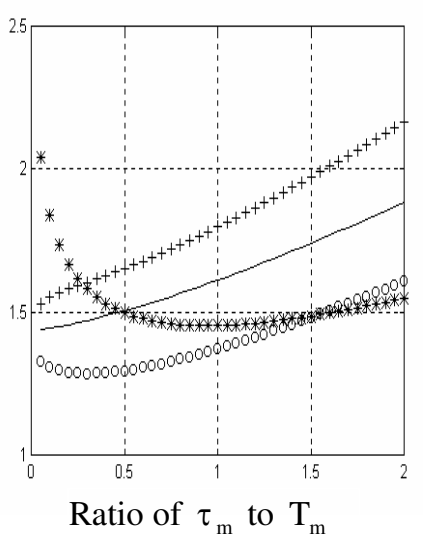

Figure 2: Phase margin

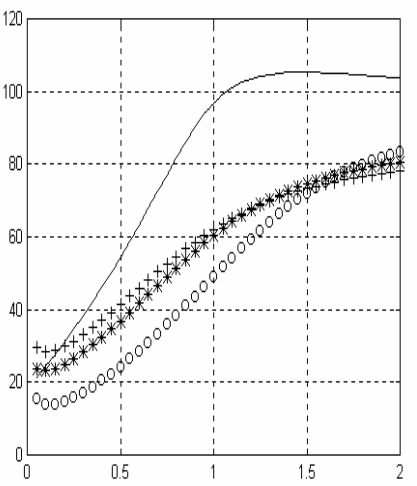

Ratio of $\tau_{\mathrm{m}}$ to $\mathrm{T}_{\mathrm{m}}$

Figure 5: Phase margin

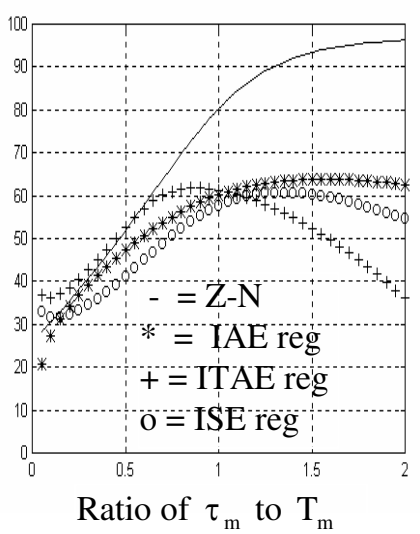

Figure 3: Maximum sensitivity

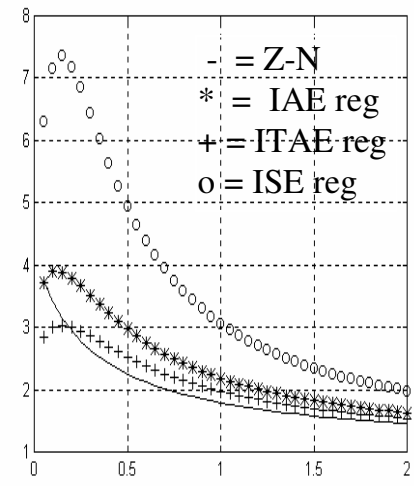

Ratio of $\tau_{\mathrm{m}}$ to $\mathrm{T}_{\mathrm{m}}$

Figure 6: Maximum sensitivity

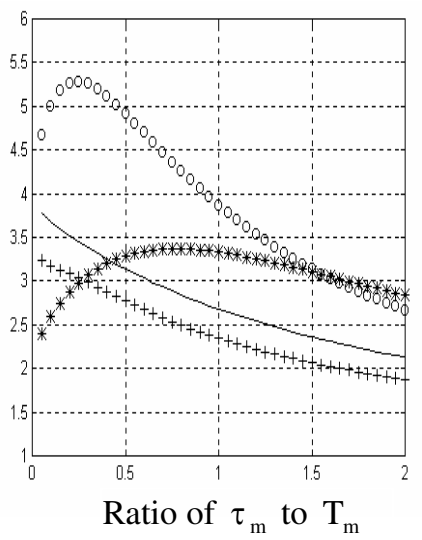

It is clear that the gain margin is consistently less when the PID rather than the PI tuning rules are considered, for all ratios of time delay to time constant taken; the difference between the phase margins is less clear cut. This suggests that these PID tuning rules should provide a greater degree of performance than the corresponding PI tuning rules, but may be less robust. Comparing the individual tuning rules, it is striking that the ISE based tuning rules 
Proceedings of the Irish Signals and Systems Conference, Dublin Institute of Technology, Kevin St., Dublin, Ireland, June, pp. 227-234.

have the smallest gain margin and have also a small phase margin, suggesting that this is a less robust tuning strategy. The results in Figures 3 and 6 confirm these comments.

Figures 7 to 12 show how the quantities vary for the other tuning rules in Table 3; Z-N ult refers to the ultimate cycle method of Ziegler and Nichols [1], A-H refers to the method specified by Astrom and Hagglund [10] with Z-A ser and Z-A reg referring to the optimal servo and regulator methods, respectively, defined by Zhuang and Atherton [11]. Figures 7 to 9 are associated with the use of the relevant PI tuning rules, with Figures 10 to 12 associated with the use of the PID tuning rules. The results speak for themselves.

Figure 7: Gain margin

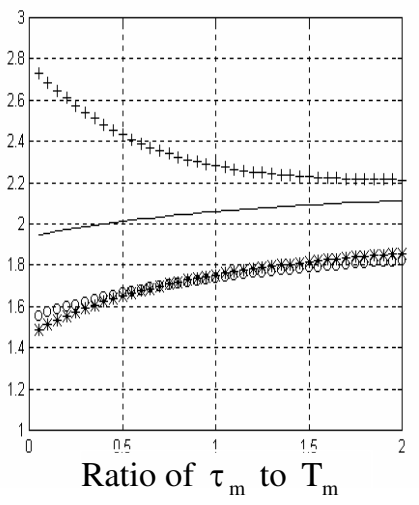

Figure 10: Gain margin

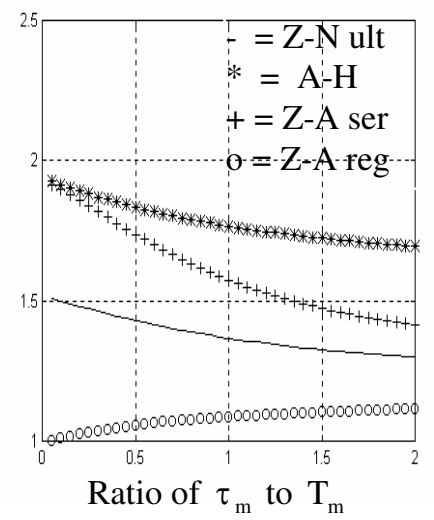

Figure 8: Phase margin

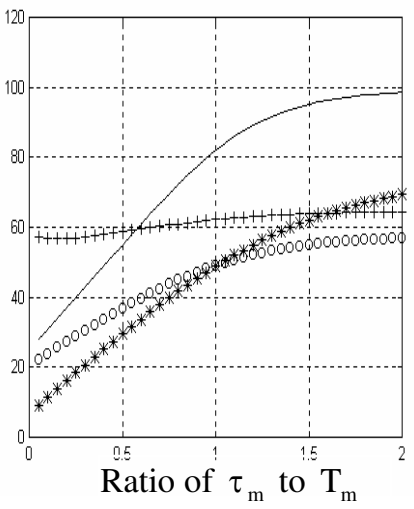

Figure 11: Phase margin

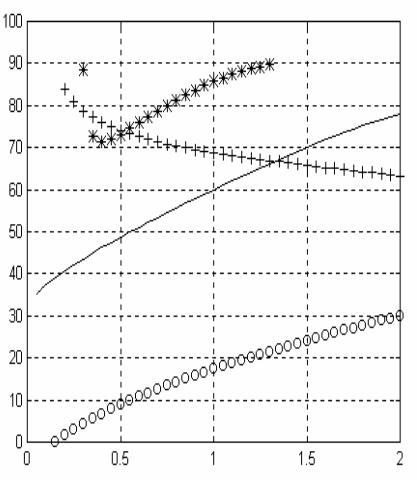

Ratio of $\tau_{\mathrm{m}}$ to $\mathrm{T}_{\mathrm{m}}$
Figure 9: Maximum sensitivity

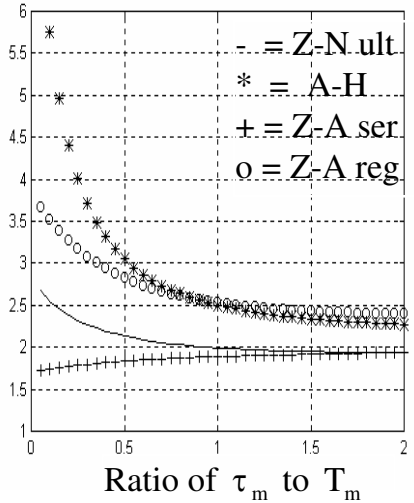

Figure 12: Maximum sensitivity

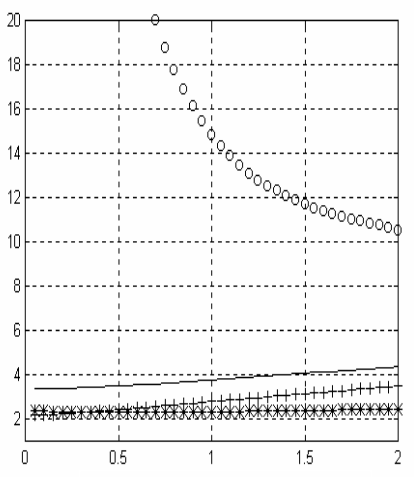

Ratio of $\tau_{\mathrm{m}}$ to $\mathrm{T}_{\mathrm{m}}$

More such results will be provided in the poster at the conference. No general conclusion can be reached as to the best tuning rule (as expected); it is interesting, though, that the full panorama of simulation results show that many tuning rules may be applied at ratios of time delay to time constant greater than that normally recommended. One example may be seen in Figures 4 to 6, where the gain and phase margin and maximum sensitivity (associated with the use of the PID tuning rule for obtaining minimum IAE in the regulator mode) tends to level out when the ratio of time delay to time constant is greater than 1; normally, the tuning rule is used when the ratio is less than 1 (Murrill [8]). On the other hand, it is clear from the same plots that there is a significant degradation of performance when using the Ziegler and Nichols [1] process reaction PID tuning rule for large ratios of time delay to time constant, which is compatible with application experience. 


\section{Conclusions}

The paper has considered the performance and robustness of a PI and PID controlled FOLPD process, with the parameters of the controllers determined by a variety of tuning rules. The original contributions of this work are as follows: (a) an expansion of the analytical approach of Ho et al. [6], [7] to determine the approximate gain and phase margin analytically under all operating conditions (b) the analytical determination of the approximate maximum sensitivity of the compensated system and (c) the application of the algorithms using a wide variety of PI and PID tuning rules. The implementation of autotuning algorithms in commercial controllers means that choice of a suitable tuning rule is an important issue; the techniques discussed allow an analytical evaluation to be performed of candidate tuning rules.

\section{References}

[1] Ziegler, J.G. and Nichols, N.B. (1942). Optimum settings for automatic controllers, Transactions of the ASME, 64, 759-768.

[2] Rovira, A.A., Murrill, P.W. and Smith, C.L. (1969). Tuning controllers for setpoint changes, Instruments and Control Systems, December, 67-69.

[3] Smith, C.A. and Corripio, A.B. (1985). Principles and practice of automatic process control, John Wiley and Sons.

[4] Hang, C.C., Lee, T.H. and Ho, W.K. (1993). Adaptive Control, Instrument Society of America.

[5] Morari, M. and Zafiriou, E. (1989). Robust process control, Prentice-Hall Inc.

[6] Ho, W.K., Hang, C.C. and Zhou, J.H. (1995). Performance and gain and phase margins of well-known PI tuning formulas, IEEE Transactions on Control Systems Technology, 3, 245248.

[7] Ho, W.K., Gan, O.P., Tay, E.B. and Ang, E.L. (1996). Performance and gain and phase margins of well-known PID tuning formulas, IEEE Transactions on Control Systems Technology, 4, 4, 473-477.

[8] Murrill, P.W. (1967). Automatic control of processes, International Textbook Co.

[9] Kaya, A. and Scheib, T.J. (1988). Tuning of PID controls of different structures, Control Engineering, 62-65.

[10] Astrom, K.J. and Hagglund, T. (1995). PID Controllers: Theory, Design and Tuning, Second Edition, Instrument Society of America.

[11] Zhuang, M. and Atherton, D.P. (1993). Automatic tuning of optimum PID controllers, IEE Proceedings, Part D, 140, 216-224. 